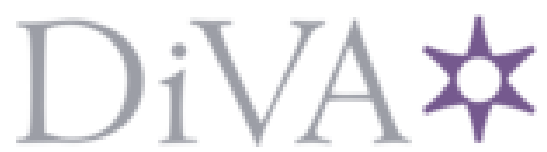

http://www.diva-portal.org

This is the published version of a paper presented at SIGCOMM.

Citation for the original published paper:

Paladi, N., Svenningsson, J., Medina, J., Arlos, P. (2019)

Protecting OpenFlow Flow Tables with Intel SGX

In: Proceedings of the ACM SIGCOMM 2019 Conference Posters and Demos (pp. 146-147). Beijing: ACM Publications

https://doi.org/10.1145/3342280.3342339

N.B. When citing this work, cite the original published paper.

Permanent link to this version:

http://urn.kb.se/resolve?urn=urn:nbn:se:bth-18654 


\section{Protecting OpenFlow Flow Tables with Intel SGX}

\author{
Nicolae Paladi \\ Lund University and RISE \\ nicolae.paladi@ri.se \\ Jorge Medina \\ New Jersey Institute of Technology \\ jmc237@njit.edu
}

\author{
Jakob Svenningsson \\ RISE \\ jakob.svenningsson@ri.se \\ Patrik Arlos \\ Blekinge Institute of Technology \\ patrik.arlos@bth.se
}

\section{CCS CONCEPTS}

- Security and privacy $\rightarrow$ Software security engineering; Security protocols; $\bullet$ Networks $\rightarrow$ Bridges and switches;

ACM Reference Format:

Nicolae Paladi, Jakob Svenningsson, Jorge Medina, and Patrik Arlos. 2019. Protecting OpenFlow Flow Tables with Intel SGX. In Proceedings of SIGCOMM '19: ACM SIGCOMM 2019 Conference, Beijing, China, August 19-23, 2019 (SIGCOMM Posters and Demos '19), 2 pages.

https://doi.org/10.1145/3342280.3342339

\section{INTRODUCTION}

Flexible and powerful control over network flows is one of the core advantages of Software-Defined Networking (SDN). Flow rules stored in switch network flow tables contain information on packet processing and routing. Flow rules are stored in memory, in a set of data structure rules, and managed by a classifier in flow tables. Network flows are a valuable asset: they contain information about the traffic patterns between the endpoints, while network tenants may be competing for the (limited) entries in flow tables [3, 4].

Commodity software switches do not currently implement confidentiality or integrity protection of flow tables. An attacker can exploit software vulnerabilities to access the switch host memory and observe or modify installed flows. Observing installed flows allows an attacker to learn security-sensitive information: topology, flow patterns between endpoints, and flow priority. Modifying installed flows allows an attacker to exploit routing loopholes and avoid certain packet steps - e.g. route around a firewall or prevent mirroring packets to an intrusion detection system.

In this demo we present OFTinSGX, an approach to protect the confidentiality and integrity of network flows installed on software switches. Our approach is based on decomposing the network switch to reduce the attack surface by isolating the OpenFlow flow tables and the flow rules from the rest of the code base. OFTinSGX allows to prevent attacks on the confidentiality and integrity of flow rules in software switches.

Permission to make digital or hard copies of all or part of this work for personal or classroom use is granted without fee provided that copies are not made or distributed for profit or commercial advantage and that copies bear this notice and the full citation on the first page. Copyrights for components of this work owned by others than ACM must be honored. Abstracting with credit is permitted. To copy otherwise, or republish, to post on servers or to redistribute to lists, requires prior specific permission and/or a fee. Request permissions from permissions@acm.org.

SIGCOMM Posters and Demos '19, August 19-23, 2019, Beijing, China

(C) 2019 Association for Computing Machinery.

ACM ISBN 978-1-4503-6886-5/19/08 ..\$15.00

https://doi.org/10.1145/3342280.3342339
Protecting the security assets of network elements is a topic of active on-going research. Jacquin proposed an architecture that used a hardware root of trust to remotely attest the integrity of virtualization hosts in SDN infrastructure [2]. Furthermore, commodity TEEs were used in case studies on securing network applications [8]. TruSDN is a framework for bootstrapping trust in an SDN infrastructure [5]. While it supports secure provisioning of switches to SGX enclaves, it was implemented with a rudimentary software switch in a SGX emulator and incurrs a significant performance overhead. Trusted Click [1] explores the feasibility of performing network processing in SGX enclaves. While none of the approaches above adress the integrity and confidentiality of OpenFlow flow tables, they can be complemented with OFTinSGX to achieve this.

Event Handler Eviction mitigates DoS attacks and overflow of OpenFlow flow table [7]. The mechanism uses two independent modules - learning module and flow checking module - that While the event handler mechanism reduces overflow of flow tables and the risk of DoS attacks, it does not provide security guarantees to the OpenFLow flow tables. OFTinSGX protects both the integrity and confidentiality of the OpenFlow flow tables, as well as the forwarding logic and eviction process. TLSonSGX protects the cryptographic material used by OvS instances to protect communication with SDN controllers [6]. The approach can be combined with OFTinSGX for wider security guarantees for OpenFlow switches.

The prototype is developed based on a modified version of Open vSwitch (OvS) version 1.11.1 and the RYU controller, version 2.8.

\section{ARCHITECTURE}

We next describe the design and implementation of OFTinSGX, a security mechanism allowing OvS to allocate its OpenFlow flow tables and forwarding logic inside enclave memory. OFTinSGX is comprised of four components: the SGX OpenFlow tables, the SGX rule mechanism, the SGX Eviction Component and the SGX Tables $d p i f$. We illustrate the interaction of these components in Figure 1.

\subsection{OFTinSGX Components}

SGX OpenFlowtables implement OpenFlow flow tables running in the enclave memory. SGX OpenFlowtables host the forwarding logic to match classification rules for unknown incoming flows. Classification rules are translated to instructions installed in the data path.

The SGX rule mechanism is an interface that implements the logic to map rules allocated in untrusted memory to classification rules in the enclave for matches in the SGX OpenFlowtables. It ensures that only rules created by an authenticated SDN controller are used to 


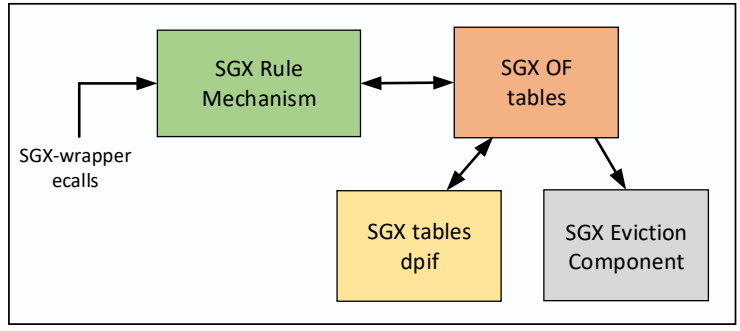

Figure 1: OFTinSGX architecture

install entries in the data path. The SGX rule mechanism translates the SGX-wrapper calls to direct operations on the OpenFlow flow tables. It is structured as a hash map table (SGX_cls_table), enclosing SGX rules (SGX_cls_rule). We used the hmap OvS library to create and manipulate the SGX hash map table.

The SGX Eviction Component implements the rule eviction process, which based on a given criteria (kept in the OpenFlow flow tables) removes a classification rule from the classifier when a table reaches the maximum number of allowed flows. In addition, the SGX Eviction Component contains the eviction groups (struct eviction_group).

Modifying any rule in the classifier requires flow validation or an update of the instantiated flow in the corresponding facet. This is necessary to monitor entries in the data path and collect statistics according to the modified flow. The dpif SGX tables implement struct table_dpif to maintain updated information about the existing rules in the OpenFlow flow tables. OvS uses dpif SGX tables when performing update routines to the facets and to maintain an updated datapath cache.

\subsection{Workflow Summary}

Prior to sending any OpenFlow request to OvS, a remote SDN controller attests the integrity of the enclave where the OpenFlow flow tables are hosted (1). Upon a successful attestation, the controller sends OpenFlow requests to operate the OpenFlow flow tables (2). If the requested operation is a rule addition, a rule is first allocated in untrusted memory and an SGX rule containing a classification rule is allocated in the enclave memory. The SGX Rule Mechanism receives the rule operation requests and handles the requests inside the enclave by calling the services of the respective processing component (3): lookup, eviction or flow revalidation.

The SGX Rule Mechanism contacts the OpenFlow flow tables when the data path does not find instructions to handle an incoming packet. In this scenario, an upcall request for a rule lookup is passed to user space, containing the incoming packet the data path cannot handle(4). If a matching classification rule is available, the SGX Rule mechanism returns the address of the corresponding untrusted rule and the fundamental information, about the matching classification rule, to install an entry in the data path cache for the incoming packet (i.e., a flow and a mask) (5). The entry in the data path is installed once the rule, allocated in untrusted memory, has been identified and the actions to be executed on that packet provided (6).
The OpenFlow flow tables deployed in SGX enclaves are protected from malicious tampering (7).

The SDN controller maintains the hash measurement of the enclave and the private key used to signed the enclave, and later uses them to establish an authenticated channel with the enclave. As a result, only an authenticated SDN controller can operate on the OpenFlow flow tables.

\section{USE CASES AND DEMONSTRATION}

The approach is based on three scenarios introduced below: rule lookup, flow addition, and flow deletion ${ }^{1}$. Rule lookup is invoked when the user space contacts OpenFlow flow tables for a matching classification rule. Flow Addition is invoked when adding new flows, contains a large number of ported functions interacting with OpenFlow flow tables. Flow Deletion flows installed in OpenFlow flow tables are removed using the del-flows command in ovs-ofctl. This operation is implemented in two steps: collecting flows and deleting flows from the OpenFlow flow tables.

\section{FUTURE WORK}

Isolating only the contents of OpenFlow flow tables does not address all security risks, since a classifier keeps only references of the classification rules. Classification rules are allocated in the struct rule, which is pointed by a struct rule_dpif, in untrusted memory. We limited the implementation scope to porting the contents of the classification rule to enclave memory and leave porting classification rules for future work. Ongoing work is focused on improving the performance of rule addition and eviction and will be reported in an upcoming publication. Future work will extend the security features of the prototype to support network slicing.

\section{ACKNOWLEDGEMENTS}

This work was financially supported in part by the Swedish Foundation for Strategic Research, grant RIT17-0035 and EU H2020 project ASCLEPIOS, grant 826093.

\section{REFERENCES}

[1] Michael Coughlin, Eric Keller, and Eric Wustrow. 2017. Trusted Click: Overcoming Security Issues of NFV in the Cloud. In SDN-NFVSec '17. ACM, 31-36.

[2] L. Jacquin, A. L. Shaw, and C. Dalton. 2015. Towards trusted software-defined networks using a hardware-based Integrity Measurement Architecture. In Proc. 1st IEEE Conf. Network Softwarization (NetSoft'15).

[3] Teemu Koponen, Keith Amidon, Peter Balland, Martín Casado, Anupam Chanda, Bryan Fulton, Igor Ganichev, Jesse Gross, Paul Ingram, Ethan J Jackson, et al. 2014. Network Virtualization in Multi-tenant Datacenters.. In NSDI, Vol. 14. 203-216.

[4] J. Matias, J. Garay, N. Toledo, J. Unzilla, and E. Jacob. 2015. Toward an SDNenabled NFV architecture. IEEE Communications Magazine 53, 4 (April 2015), 187-193. https://doi.org/10.1109/MCOM.2015.7081093

[5] Nicolae Paladi and Christian Gehrmann. 2016. TruSDN: Bootstrapping Trust in Cloud Network Infrastructure. In Proc. 12th International Conf. on Security and Privacy in Communication Networks (SecureComm'16). Springer.

[6] Nicolae Paladi, Linus Karlsson, and Khalid Elbashir. 2018. Trust Anchors in Software Defined Networks. In Computer Security, Javier Lopez, Jianying Zhou, and Miguel Soriano (Eds.). Springer International Publishing, Cham, 485-504.

[7] Ying Qian, Wanqing You, and Kai Qian. 2016. OpenFlow flow table overflow attacks and countermeasures. In 2016 European Conf. on Networks and Communications (EuCNC). 205-209. https://doi.org/10.1109/EuCNC.2016.7561033

[8] Ming-Wei Shih, Mohan Kumar, Taesoo Kim, and Ada Gavrilovska. 2016. S-NFV: Securing NFV States by Using SGX. In Proc. 2016 ACM International Workshop on Security in Software Defined Networks \& Network Function Virtualization (SDNNFV Security' 16). ACM, 45-48.

${ }^{1}$ Demo video: https://vimeo.com/217788815 